\title{
TRANSIENT RESPONSE OF AN ANTIPLANE INCLINED SUBSURFACE CRACK SUBJECTED TO DYNAMIC MOVING LOADINGS
}

\author{
CHIEN-CHING MA and LYANG-RUNG HWANG \\ Department of Mechanical Engineering, National Taiwan University, Taipei, Taiwan 10617, \\ Republic of China
}

(Received 20 July 1994; in revised form 15 May 1995)

\begin{abstract}
The transient response of a half-space containing a subsurface inclined semi-infinite crack excited by a dynamic antiplane loading on the surface of the half-space has been investigated to gain insight into the phenomenon of the interaction of stress waves with material defects. The dynamic load is suddenly applied and then it moves at a constant speed along the free surface. The solutions are derived for all load speeds (subsonic and supersonic) and are determined by superposition of a proposed fundamental solution in the Laplace transform domain. The fundamental problem to be considered is the exponentially distributed traction in Laplace transform domain applied on crack faces. The method of analysis is based on integral transform techniques and the Wiener-Hopf technique. The exact closed-form transient full-field solutions of stresses and displacement are obtained in compact formulations. These solutions are valid for an infinite length of time and have accounted for the contributions of incident, reflected and diffracted waves. Numerical results of the transient stresses for the interior of the half-space are obtained and the results of the limit case of zero load speed are compared with the corresponding static values. Copyright (C) 1996 Elsevier Science Ltd.
\end{abstract}

\section{INTRODUCTION}

The difficulty in determining the transient stress field in a cracked elastic body subjected to dynamic loading is well known. A considerable amount of research has been directed towards the solution of problems involving the interaction of stress waves with cracks and boundaries to improve understanding of the behavior of material failure under dynamic loading. The early solutions of waves generated by moving loads were confined to the steady state response of the medium. Transient response due to a concentrated force moving in an infinite medium was determined by Payton (1964) and the transient solution for a force moving in a half-space was solved by Gakenheimer and Miklowitz (1969). In conventional studies of a semi-infinite crack in an unbounded medium subjected to a spatially uniform crack face traction, the complete solution is usually obtained by integral transform methods together with direct application of the Wiener-Hopf technique (Noble, 1958) and the Cagniard-de Hoop method (de Hoop, 1958) of Laplace inversion. If the crack face traction distribution is spatially nonuniform and a characteristic length is introduced, then this procedure using integral transformation methods does not apply. The problem of an elastic solid containing a semi-infinite crack subjected to concentrated impact loading on the faces of the crack has been studied by Freund (1974). He proposed a fundamental solution arising from an edge dislocation climbing along the line ahead of the crack tip with a constant speed to overcome these difficulties of the case with a characteristic length. The solution can be constructed by taking an integration over a climbing dislocation of different moving velocity along the crack tip line. Basing their procedures on this method, Brock $(1982,1984)$ and Brock et al. $(1985)$, and Ma and Hou $(1990,1991)$ have recently analyzed a series of problems involving a semi-infinite crack subjected to dynamic impact loadings. Lee and Freund (1990) analyzed fracture initiation of an edge-cracked plate subjected to an asymmetric impact.

Whenever dynamic loading is applied to a body with an internal crack, the resulting stress waves may initiate crack growth. Few solutions for a cracked elastic solid subjected to dynamic loading are available. Exact transient closed-form solutions for a stationary 


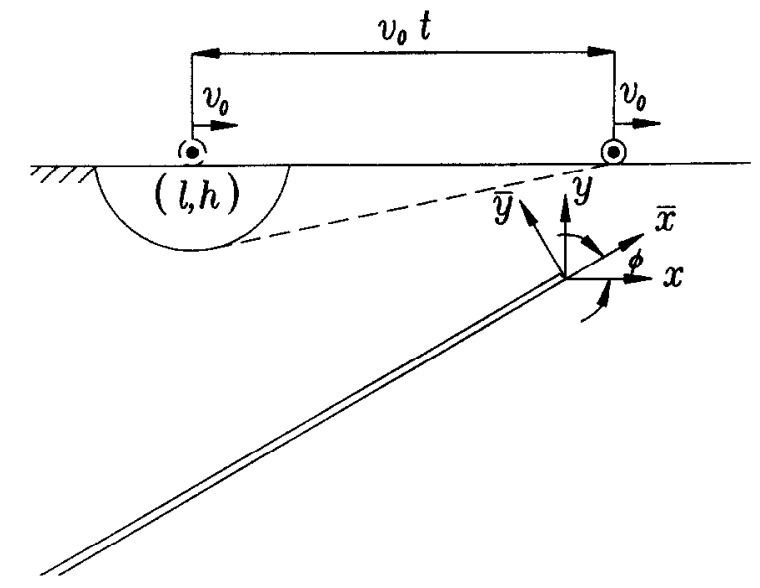

Fig. 1. Configuration, coordinate systems of a subsurface inclined crack subjected to dynamic loading moving with supersonic speed on the half-space.

semi-infinite crack subjected to a suddenly applied dynamic body force in an unbounded medium have been obtained by Tsai and Ma (1992) for the in-plane case, and by Ma and Chen (1993) and Brock (1986) for the antiplane case. The problem to be considered in this study is the transient response of an elastic half-plane, with an inclined subsurface crack extending from infinity to a location near the half-plane surface. The cracked half-plane is subjected to a dynamic antiplane loading moving with constant speed on the half-plane surface as shown in Fig. 1, the moving speed can be either subsonic or supersonic. To the author's knowledge, most solutions for scattering of elastic waves by a crack, located in the immediate vicinity of a free surface, are given for incident plane time-harmonic waves [e.g. Achenbach and Brind (1981) and Brind and Achenbach (1981)]. The only available transient analysis is presented by Ma and Chen (1994) who consider the special case of applying dynamic loading with zero moving speed. The propagation of stress waves through a semi-infinite homogeneous medium with a half-plane boundary, which is due to applying dynamic loading on the boundary, is not a difficult subject. A pre-existing fault inside the medium would disturb the propagation waves and make the theoretical analysis much more difficult than in an homogeneous medium. The transient response of the cracked half-plane is analyzed as the superposition of the fields in an uncracked half-plane and the fields generated by appropriate surface tractions on the faces of the crack in the cracked halfplane. In analyzing this problem, the reflections and diffractions of stress waves by the halfplane boundary and by the crack will generate an infinite number of waves, that must be taken into account. This problem involves a characteristic length which makes a direct solution by standard techniques difficult. A new fundamental solution is used for overcoming these difficulties. This alternative fundamental solution is successfully applied towards solving the problem and is to be demonstrated as an efficient methodology. The final formulations for stresses and displacement are expressed explicitly and the dynamic effect of each wave is presented in a closed form. The results are valid for infinite waves that are scattered from the crack tip and reflected by the half-plane boundary. Numerical results are presented for the shear stresses at points of observation in the interior of the body and for the loading moving with various speeds along the half-plane free surface.

\section{REQUIRED FUNDAMENTAL SOLUTIONS}

As usual in problems of the type considered here, superposition of solutions plays a significant role. The solutions of the problem considered in this study can be determined by superposition of the following problems. Problem A treats the dynamic concentrated force moving with constant velocity on a half-plane medium without a crack, which induces a traction on the planes that will eventually define the semi-infinite crack faces. In problem B, an infinite body containing a semi-infinite crack is considered in which the crack faces are subjected to tractions which are equal and opposite to those on the corresponding 
planes in problem A. Problem C considers a half-plane free surface subjected to the waves which are generated by the crack in problcm $B$.

The solution for problem $\mathrm{A}$ is well known and problem $\mathrm{C}$ can be solved by using the image method. Hence, problem $B$ in the above mentioned three fundamental problems is the only one which needs careful analysis. Reflected and diffracted waves are generated by the semi-infinite crack to eliminate the stress induced by incident waves on the traction-free boundaries of crack faces. For most of the dynamic problems, the incident waves can be represented in an exponential functional form in the Laplace transform domain of time. The exponentially distributed loading acting on the crack faces can be analyzed, giving rise to a very useful result called the fundamental solution for the problem. Then, the corresponding field quantities can be determined directly by superposition over this fundamental solution. The superposition scheme proposed in this study, unlike the usual superposition methods which are performed in the time domain, is performed in the Laplace transform domain.

The solution for applying an exponentially distributed loading on the crack faces in the Laplace transform domain will be referred to as the fundamental solution. The fundamental problem can be viewed as a half-plane problem with the material occupying the region $y \geqslant 0$, subject to the following boundary conditions

$$
\begin{array}{cc}
\bar{\tau}_{y z}(x, 0, p)=\mathrm{e}^{p \eta x} & -\infty<x<0, \\
\bar{w}(x, 0, p)=0 & 0<x<\infty,
\end{array}
$$

where $p$ is the Laplace transform parameter and $\eta$ is a constant. The overbar symbol is used for denoting the transform on time $t$. The governing equation can be represented by the following two-dimensional wave equation

$$
\frac{\partial^{2} w}{\partial x^{2}}+\frac{\partial^{2} w}{\partial y^{2}}=b^{2} \frac{\partial^{2} w}{\partial t^{2}}
$$

where $b$ is the slowness of the transverse wave given by

$$
b=1 / v_{\mathrm{s}}=\sqrt{\rho / \mu},
$$

in which $w(x, y, t)$ is the displacement normal to the $x y$-plane; $v_{\mathrm{s}}$ is the shear wave speed, $\mu$ and $\rho$ are the respective shear modulus and mass density of the material. The non-vanishing shear stresses are

$$
\tau_{y z}=\mu \frac{\partial w}{\partial y}, \quad \tau_{x z}=\mu \frac{\partial w}{\partial x} .
$$

This problem can be solved by the application of integral transforms. The solution is obtained by applying the one-sided Laplace transform over time, the two-sided Laplace transform over $x$ under the restriction of $\operatorname{Re}(\eta)>\operatorname{Re}(\lambda)$, and finally implementing the Wiener-Hopf technique. The solutions of stresses and displacement expressed in the Laplace transform domain for the boundary conditions (1) and (2) are

$$
\begin{gathered}
\tau_{y z}^{*}(\lambda, y, p)=\frac{(b+\lambda)^{1 / 2} \mathrm{e}^{-p x y}}{p(b+\eta)^{1 / 2}(\eta-\lambda)}, \\
\bar{\tau}_{x z}^{*}(\lambda, y, p)=\frac{\lambda \mathrm{e}^{-p \alpha y}}{p(b+\eta)^{1 / 2}(\eta-\lambda)(b-\lambda)^{1 / 2}}, \\
\bar{w}^{*}(\lambda, y, p)=\frac{\mathrm{e}^{-p \alpha y}}{\mu p^{2}(b+\eta)^{1 / 2}(\eta-\lambda)(b-\lambda)^{1 / 2}} .
\end{gathered}
$$


The inversion formula for the two-sided Laplace transform (Achenbach, 1973) leads to the following expressions

$$
\begin{gathered}
\bar{\tau}_{y z}(x, y, p)=\frac{1}{2 \pi i} \int_{\Gamma_{i}} \frac{(b+\lambda)^{1 / 2} \mathrm{e}^{-p(\alpha y-\lambda x)}}{(b+\eta)^{1 / 2}(\eta-\lambda)} \mathrm{d} \lambda, \\
\bar{\tau}_{x z}(x, y, p)=-\frac{1}{2 \pi i} \int_{\Gamma_{\lambda}} \frac{\lambda \mathrm{e}^{-p(\alpha y-\lambda x)}}{(b+\eta)^{1 / 2}(\eta-\lambda)(b-\lambda)^{1 / 2}} \mathrm{~d} \lambda, \\
\bar{w}(x, y, p)=-\frac{1}{2 \pi i} \int_{\Gamma_{\lambda}} \frac{\mathrm{e}^{-p(\alpha y-\lambda x)}}{\mu p(b+\eta)^{1 / 2}(\eta-\lambda)(b-\lambda)^{1 / 2}} \mathrm{~d} \lambda,
\end{gathered}
$$

where $\Gamma_{\lambda}$ is a suitable path of integration in the complex $\lambda$-plane and

$$
\alpha=\left(b^{2}-\lambda^{2}\right)^{1 / 2}
$$

The fundamental solutions presented in eqns (5)-(7) will be used to construct the reflected and diffracted waves generated by the crack in the following analysis.

\section{EXACT TRANSIENT FULL-FIELD ANALYSIS}

The investigation of a subsurface crack subjected to dynamic moving loading is an important topic in material failure analysis. The problem considered here is an inclined semi-infinite crack located under the surface of a half-plane (Fig. 1). Having two coordinate systems is convenient for the following analysis since the surface of the half-plane is not parallel to the crack faces. The origins of the two coordinate systems $(\bar{x}, \bar{y})$ and $(x, y)$ are both located at the crack tip as shown in Fig. 1. The planar crack lies in the plane $\bar{y}=0$, $\bar{x}<0$ and the inclined angle of the crack is $\phi$. The coordinate transforms and stress relations between these two systems are

$$
\begin{gathered}
\bar{x}=x \cos \phi+y \sin \phi, \\
\bar{y}=-x \sin \phi+y \cos \phi, \\
\tau_{x z}=\tau_{\overline{x z}} \cos \phi-\tau_{\overline{y z}} \sin \phi, \\
\tau_{y z}=\tau_{\overline{x z}} \sin \phi+\tau_{\overline{y z}} \cos \phi .
\end{gathered}
$$

The problem to be considered in this study has a characteristic length. A direct attempt towards solving this problem by transform and Wiener-Hopf techniques is not applicable. The transient elastodynamic problem will be solved by superposition of the fundamental solutions obtained in the previous section in the Laplace transform domain. The transient solutions are composed of an incident field, reflected field and diffracted field, which will be denoted by superscripts of $i, r$ and $d$ for the case of subsonic moving speed and additional superscripts of $I, R$ and $D$ will be used for the supersonic case, respectively. The incident wave is the response for applying a dynamic loading that moves along an uncracked halfplane with constant velocity. The reflected and diffracted waves are generated through application of an opposite traction at the crack surface thus eliminating the stress induced by the incident wave.

Consider a half-plane medium which is stress-free and at rest. At time $t=0$, an antiplane concentrated dynamic load of magnitude $Q$ is applied at the free surface of a halfplane at position $(l, h)$. For $t>0$, this concentrated load travels on the surface along the positive $x$-axis with a constant speed $v_{0}$. The time dependence of the loading is represented 
by the Heaviside step function $H(t)$. The incident fields of displacement and shear stresses in the Laplace transform domain can be obtained as follows

$$
\begin{gathered}
\bar{w}^{i}(x, y, p)=\frac{1}{2 \pi i} \int_{\Gamma_{\lambda}} \frac{Q}{\mu p \alpha\left(1+\lambda v_{0}\right)} \mathrm{e}^{p[\alpha(y-h)+\lambda(x-)] \mathrm{d} \lambda}, \\
\bar{\tau}_{y z}^{i}(x, y, p)=\frac{1}{2 \pi i} \int_{\Gamma_{\lambda}} \frac{Q}{1+\lambda v_{0}} \mathrm{e}^{p[\alpha(y-h)+\lambda(x-h)]} \mathrm{d} \lambda, \\
\bar{\tau}_{x z}^{i}(x, y, p)=\frac{1}{2 \pi i} \int_{\Gamma_{\lambda}} \frac{\lambda Q}{\alpha\left(1+\lambda v_{0}\right)} \mathrm{e}^{p[\alpha(y-h)+\lambda(x-h)]} \mathrm{d} \lambda,
\end{gathered}
$$

where $\Gamma_{\lambda}$ is a suitable path of integration in the complex $\lambda$-plane. The Laplace transform is inverted by a modification of Cagniard's (1962) technique due to de Hoop (1958). For this technique eqns (12)-(14) are converted into the Laplace transform of a known function, so that the Laplace transform can be inverted by inspection. In the subsequent calculations $p$ is assumed to be a real and positive number. The transient solution has different forms depending on the speed of the moving load relative to the shear wave speed. In particular, the terms supersonic and subsonic refer to the cases when the load speed is greater than the shear wave speed $\left(v_{0}>v_{\mathrm{s}}\right)$ or less than the shear wave speed $\left(v_{0}<v_{\mathrm{s}}\right)$, respectively.

The detailed inversion of Laplace transform for (12)-(14) to time domain can be found in a paper by Ma and Chen (1994), and the results are

$$
\begin{aligned}
\tau_{y z}^{i}(x, y, t)= & \frac{Q\left(-v_{0} b^{2} r^{2} \sin \theta \cos \theta+r t \sin \theta\right)}{\pi\left(t^{2}-b^{2} r^{2}\right)^{1 / 2}\left(r^{2}-2 r v_{0} t \cos \theta-v_{0}^{2} b^{2} r^{2} \sin ^{2} \theta+v_{0}^{2} t^{2}\right)} H(t-b r) \\
& +\frac{Q}{v_{0}} \delta\left[t-c(x-l)+\sqrt{b^{2}-c^{2}}(y-h)\right] H\left(v_{0}-v_{\mathrm{s}}\right) H(b \cos \theta-c), \\
\tau_{x z}^{i}(x, y, t)= & \frac{Q\left(v_{0} t^{2}-v_{0} b^{2} r^{2} \sin ^{2} \theta-r t \cos \theta\right)}{\pi\left(t^{2}-b^{2} r^{2}\right)^{1 / 2}\left(r^{2}-2 r v_{0} t \cos \theta-v_{0}^{2} b^{2} r^{2} \sin ^{2} \theta+v_{0}^{2} t^{2}\right)} H(t-b r) \\
& -\frac{Q}{v_{0} \sqrt{b^{2} v_{0}^{2}-1}} \delta\left[t-c(x-l)+\sqrt{b^{2}-c^{2}}(y-h)\right] H\left(v_{0}-v_{\mathrm{s}}\right) H(b \cos \theta-c), \\
w^{i}(x, y, t)= & \frac{Q}{\pi \mu} \int_{b r}^{t} \frac{\left(r^{2}-r v_{0} t \cos \theta\right)}{\left(t^{2}-b^{2} r^{2}\right)^{1 / 2}\left(r^{2}-2 r v_{0} t \cos \theta-v_{0}^{2} b^{2} r^{2} \sin ^{2} \theta+v_{0}^{2} t^{2}\right)} \mathrm{d} t \\
& +\frac{Q}{\mu \sqrt{b^{2} v_{0}^{2}-1}} H\left[t-c(x-l)+\sqrt{b^{2}-c^{2}}(y-h)\right] H\left(v_{0}-v_{\mathrm{s}}\right) H(b \cos \theta-c),
\end{aligned}
$$

where

$$
r=\left[(x-l)^{2}+(y-h)^{2}\right]^{1 / 2}, \quad \theta=\cos ^{-1}\left(\frac{x-l}{r}\right)
$$

and $c=1 / v_{0}$ is the slowness of the speed of the moving load. The symbols $\delta(\cdot)$ and $H(\cdot)$ represent the Dirac delta function and the unit step function, respectively. The contribution of the second term shown in (15)-(17) only exists for the supersonic case and represents a plane wave. For the subsonic case, only the first term is left in the transient solution expressed in (15)-(17).

The incident wave will induce a stress field $\tau_{y z}^{i}(\bar{x}, 0, p)$ along the crack face, conveniently expressed in the $\bar{x}-\bar{y}$ coordinate system as 


$$
\bar{\tau}_{\overline{y z}}^{i}(\bar{x}, 0, p)=\frac{1}{2 \pi i} \int_{\Gamma_{i}} \frac{Q}{\left(1+\lambda v_{0} \cos \phi-\alpha v_{0} \sin \phi\right)} \mathrm{e}^{-p \alpha h_{0}+p \lambda\left(\bar{x}-l_{0}\right)} \mathrm{d} \lambda,
$$

where

$$
l_{0}=l \cos \phi+h \sin \phi, \quad h_{0}=-l \sin \phi+h \cos \phi .
$$

The applied traction on the crack face, in order to eliminate the stress induced by the incident wave as indicated in eqn (18), has the functional form $\mathrm{e}^{p \lambda \varepsilon}$. Since the solutions of applying traction $e^{p \eta x}$ on crack faces have been solved in Section 2 , the reflected and diffracted fields generated from the inclined crack can be constructed by superimposing the incident wave traction that is equal and opposite to eqn (18). When we combine eqns (5) and (18), the solution of the first reflected and diffracted waves for $\bar{\tau}_{\overline{y z}}$ and $\bar{\tau}_{\overline{x z}}$ in the Laplace transform domain can be expressed as follows :

$$
\begin{aligned}
& \bar{\tau}_{\overline{y z}}^{d+r}(\bar{x}, \bar{y}, p)=\frac{Q}{4 \pi^{2}} \int_{\Gamma_{\eta_{1}}} \int_{\Gamma_{\eta_{2}}} \frac{G\left(\eta_{1}, \eta_{2}\right)}{\left[1+v_{0}\left(\eta_{1} \cos \phi-\alpha_{1} \sin \phi\right)\right]} \mathrm{e}^{-p\left(\alpha_{1} h_{0}+\eta_{1} l_{0}\right)} \mathrm{e}^{-p\left(\alpha_{2} \bar{y}-\eta_{2} \bar{x}\right)} \mathrm{d} \eta_{2} \mathrm{~d} \eta_{1}, \\
& \bar{\tau}_{\bar{x}} \frac{d+r}{(\bar{x}, \bar{y}, p)}=-\frac{Q}{4 \pi^{2}} \int_{\Gamma_{\eta_{1}}} \int_{\Gamma_{\eta_{2}}} \frac{\eta_{2}}{\alpha_{2}} \frac{G\left(\eta_{1}, \eta_{2}\right)}{\left[1+v_{0}\left(\eta_{1} \cos \phi-\alpha_{1} \sin \phi\right)\right]} \mathrm{e}^{-p\left(\alpha_{1} h_{0}+\eta_{1} l_{0}\right)} \mathrm{e}^{-p\left(\alpha_{2} \bar{y}-\eta_{2} \bar{x}\right)} \mathrm{d} \eta_{2} \mathrm{~d} \eta_{1},
\end{aligned}
$$

where

$$
\begin{gathered}
G\left(\eta_{1}, \eta_{2}\right)=\frac{\left(b+\eta_{2}\right)^{1 / 2}}{\left(b+\eta_{1}\right)^{1 / 2}\left(\eta_{1}-\eta_{2}\right)}, \\
\alpha_{1}=\left(b^{2}-\eta_{1}^{2}\right)^{1 / 2}, \quad \alpha_{2}=\left(b^{2}-\eta_{2}^{2}\right)^{1 / 2} .
\end{gathered}
$$

The above presented solutions for stresses are expressed in the $\bar{x}-\bar{y}$ coordinate system. If the solutions are expressed in the $x-y$ coordinate system, then a small modification should be made to account for the effect of coordinate transformation, and the result for $\bar{\tau}_{y z}$ is

$$
\tau_{y z}^{d+r}(x, y, p)=\frac{Q}{4 \pi^{2}} \int_{\Gamma_{\eta_{1}}} \int_{\Gamma_{\eta_{2}}} \frac{K_{1}\left(\eta_{2}\right) G\left(\eta_{1}, \eta_{2}\right)}{\left[1+v_{0}\left(\eta_{1} \cos \phi-\alpha_{1} \sin \phi\right)\right]} \mathrm{e}^{-p\left(\alpha_{1} h_{0}+\eta_{1} l_{0}\right)} \mathrm{e}^{-p\left(\alpha_{2} \bar{y}-\eta_{2} \bar{x}\right)} \mathrm{d} \eta_{2} \mathrm{~d} \eta_{1},
$$

where

$$
K_{1}\left(\eta_{2}\right)=\cos \phi-\frac{\eta_{2}}{\alpha_{2}} \sin \phi
$$

This equation constitutes a double inversion integral where the paths $\Gamma_{\eta_{1}}$ and $\Gamma_{\eta_{2}}$ refer to Laplace inversion contours in the $\eta_{1}$-plane and the $\eta_{2}$-plane, respectively. The inverse transformation is carried out here by the Cagniard-de Hoop technique. Cagniard contours are introduced here in both the $\eta_{1}$-and $\eta_{2}$-planes by setting

$$
\begin{gathered}
\alpha_{1} h_{0}+\eta_{1} l_{0}=t_{1}, \\
\alpha_{2} \bar{y}-\eta_{2} \bar{x}=t_{2} .
\end{gathered}
$$

Equations (20) and (21) can be solved for $\eta_{1}$ and $\eta_{2}$ to yield 


$$
\begin{aligned}
& \eta_{1}^{ \pm}=\frac{t_{1} \cos \Theta_{0}}{R_{0}} \pm i \frac{\sin \Theta_{0}}{R_{0}}\left(t_{1}^{2}-b^{2} R_{0}^{2}\right)^{1 / 2}, \\
& \eta_{2}^{ \pm}=-\frac{t_{2} \cos \psi}{d} \pm i \frac{\sin \psi}{d}\left(t_{2}^{2}-b^{2} d^{2}\right)^{1 / 2},
\end{aligned}
$$

where $\left(R_{0}, \Theta_{0}\right)$ and $(d, \psi)$ are the respective polar coordinates of the source point and field point, and

$$
\begin{aligned}
R_{0} & =\left(l_{0}^{2}+h_{0}^{2}\right)^{1 / 2}, \quad \Theta_{0}=\cos ^{-1}\left(l_{0} / R_{0}\right), \\
d & =\left(\bar{x}^{2}+\bar{y}^{2}\right)^{1 / 2}, \quad \psi=\cos ^{-1}(\bar{x} / d) .
\end{aligned}
$$

In the $\eta_{1}$-plane (or $\eta_{2}$-plane), eqn (22) [or (23)] describes a hyperbola which is denoted as the Cagniard contour. The $\eta_{1}$ - and $\eta_{2}$-integrations are then shifted on to Cagniard contours along which $t_{1}$ and $t_{2}$ are both real and positive. The two Cagniard contours must be superimposed in this technique for different locations of source and field points. Continuing from this, the reflected and diffracted waves generated from the crack can be automatically constructed. Because $G\left(\eta_{1}, \eta_{2}\right)$ possesses a pole at $\eta_{1}=\eta_{2}$, the contribution of the pole has to be taken into account in the change of integral paths from $\eta_{1}$ to $t_{1}$ and $\eta_{2}$ to $t_{2}$. Recall from eqn (19) that a pole term arises, representing the reflected waves. The contribution of this pole represents the reflected $r$ wave generated from the crack surface by the incident cylindrical wave. The reflected $r$ wave can be shown to pass the region for $\psi>\pi-\Theta_{v}$. By using the Cagniard-de Hoop method of Laplace inversion, reflected field in time domain is obtained in a simple closed form as follows

$$
\tau_{y z}^{r}(x, y, t)=\frac{Q\left[v_{0} t^{2} \sin 2 \phi+v_{0} b^{2} r_{1}^{2} \sin \left(\theta_{1}-2 \phi\right) \cos \theta_{1}-r_{1} t \sin \theta_{1}\right] H\left(t-b r_{1}\right)}{\pi\left(t^{2}-b^{2} r_{1}^{2}\right)^{1 / 2}\left[\left(r_{1}^{2}-2 r_{1} v_{0} t \cos \left(\theta_{1}-2 \phi\right)-v_{0}^{2} b^{2} r_{1}^{2} \sin ^{2}\left(\theta_{1}-2 \phi\right)+v_{0}^{2} t^{2}\right]\right.},
$$

where

$$
\begin{aligned}
& r_{1}=\left[\left(x-l_{1}\right)^{2}+\left(y-h_{1}\right)^{2}\right]^{1 / 2}, \quad \theta_{1}=\cos ^{-1}\left(\frac{x-l_{1}}{r_{1}}\right), \\
& l_{1}=l \cos (2 \phi)+h \sin (2 \phi), \quad h_{1}=l \sin (2 \phi)-h \cos (2 \phi) .
\end{aligned}
$$

The diffracted $d$ wave generated from the crack tip by the incident cylindrical wave is considered next. Recalling that $p$ is real and positive, the $\eta_{1}$ - and $\eta_{2}$-integrations are shifted on to Cagniard contours. The stress $\tau_{y z}^{d}$ induced by the diffracted wave from the crack tip is found to be

$$
\tau_{y z}^{d}(x, y, t)=\frac{Q}{2 \pi^{2}} \int_{b R_{0}}^{t-h d} \operatorname{Re}\left[K_{1}\left(\eta_{2}^{+}\right)\left(F\left(\eta_{1}^{+}, \eta_{2}^{+}\right) \frac{\partial \eta_{1}^{+}}{\partial t_{1}} \frac{\partial \eta_{2}^{+}}{\partial t_{2}}-F\left(\eta_{1}^{-}, \eta_{2}^{+}\right) \frac{\partial \eta_{1}^{-}}{\partial t_{1}} \frac{\partial \eta_{2}^{+}}{\partial t_{2}}\right)\right] \mathrm{d} t_{1}
$$

where

$$
F\left(\eta_{1}, \eta_{2}\right)=\frac{G\left(\eta_{1}, \eta_{2}\right)}{1+v_{0}\left(\eta_{1} \cos \phi-\alpha_{1} \sin \phi\right)}, t_{2}=t-t_{1}
$$

For the supersonic case $\left(v_{0}>v_{\mathrm{s}}\right)$, the incident wave contains an additional plane wave and this plane wave will also be reflected and diffracted from the semi-infinite crack. The 


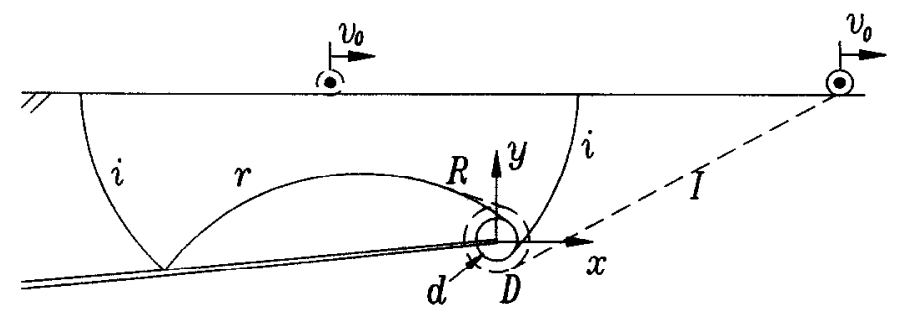

Fig. 2. Wave fronts for reflected and diffracted waves generated from the inclined crack by incident plane and cylindrical waves.

configuration of the wave fronts for the supersonic case is shown in Fig. 2. The reflected wave ( $R$ wave) and diffracted wave ( $D$ wave) generated from the crack by the incident plane wave are constructed from the contribution of the pole [i.e. $1+v_{0}\left(\eta_{1} \cos \right.$ $\left.\phi-\alpha_{1} \sin \phi\right)=0$ ] shown in eqn (19), which is $\eta_{1}=-c \cos \phi+\sqrt{b^{2}-c^{2}} \sin \phi$. The results are expressed as follows

$$
\begin{aligned}
\tau_{y z}^{R}(x, y, t)= & -\frac{Q}{v_{0}} \frac{\sqrt{b^{2} v_{0}^{2}-1} \cos 2 \phi+\sin 2 \phi}{\sqrt{b^{2} v_{0}^{2}-1}} \delta\left[t-\sqrt{b^{2}-c^{2}}(y \cos 2 \phi-x \sin 2 \phi+h)\right. \\
\tau_{y z}^{D}(x, y, t)= & -\frac{Q}{\pi} \frac{\sqrt{b^{2} v_{0}^{2}-1} \cos \phi+\sin \phi}{\sqrt{b^{2} v_{0}^{2}-1}\left(b-c \cos \phi+\sqrt{b^{2}-c^{2}} \sin \phi\right)^{1 / 2}} \\
& \operatorname{Im}\left[\frac{K_{1}\left(\eta_{2}^{\prime}\right)\left(b+\eta_{2}^{\prime}\right)^{1 / 2}}{-\cos \phi+\sin \phi \sqrt{b^{2} v_{0}^{2}-1}-\eta_{2}^{\prime} v_{0}} \frac{\partial \eta_{2}^{\prime}}{\partial t}\right] H\left(t-b d+c l-\sqrt{b^{2}-c^{2}} h\right),
\end{aligned}
$$

wherc

$$
\eta_{2}^{\prime}=-\frac{\cos \psi}{d}\left[t+c l-\sqrt{b^{2}-c^{2}} h\right]+i \frac{\sin \psi}{d}\left[\left(t+c l-\sqrt{b^{2}-c^{2}} h\right)^{2}-b^{2} d^{2}\right]^{1 / 2}
$$

For the subsonic case, the incident cylindrical shear wave ( $i$ wave) will generate only a reflected wave ( $r$ wave) and a diffracted wave ( $d$ wave) from the subsurface crack. After some later time, these two waves will be reflected from the free half-surface and are indicated as the $r r$ wave and $d r$ wave. The solutions for $r r$ and $d r$ waves can be constructed by employing the method of images, which can be obtained from the solutions of $r$ and $d$ waves, hence the results are omitted here. The reflected $r r$ and $d r$ waves will arrive at the crack at a later time. The reflected waves ( $r r r$ and $d r r$ waves) and diffracted waves ( $r r d$ and $d r d$ waves) generated by the crack due to incidenting $r r$ and $d r$ waves can be constructed following the previously indicated analysis. For the supersonic case, additional waves should be included which are initiated by the incident plane wave. There are four waves, i.e. $D R D, D R R, R R D$ and $R R R$ waves, will be generated by the crack due to incidenting $R R$ and $D R$ waves. The correspondent configuration of the wave fronts for the supersonic case is shown in Fig. 3.

The complete full-field solutions that account for the contributions of all the reflected and diffracted waves are finally obtained explicitly. The complete transient solutions for stresses and displacement can be simplified into a form as follows

$$
\begin{aligned}
& \tau_{y z}=\sum_{i=0}^{s} \tau_{y z}^{i}+\sum_{k=0}^{n} \sum_{l=0}^{\infty} \sum_{j=0}^{m} \tau_{y z}^{j, k, l}+\left[\sum_{i=0}^{s^{\prime}} \tau_{y z}^{i^{\prime}}+\sum_{k^{\prime}=0}^{n^{\prime}} \sum_{j=0}^{m} \tau_{y z}^{j, k^{\prime}, 0}+\sum_{k^{\prime}=0}^{n^{\prime}} \sum_{l^{\prime}=1}^{\infty} \sum_{j=0}^{m} \tau_{y z}^{j, k^{\prime}, r}\right] H\left(v_{0}-v_{\mathrm{s}}\right), \\
& \tau_{x z}=\sum_{i=0}^{s} \tau_{x z}^{i}+\sum_{k=0}^{n} \sum_{l=0}^{\infty} \sum_{j=0}^{m} \tau_{x z}^{j, k, l}+\left[\sum_{i=0}^{s^{\prime}} \tau_{x z}^{i^{\prime}}+\sum_{k^{\prime}=0}^{n^{\prime}} \sum_{j=0}^{m} \tau_{x z}^{j, k^{\prime}, 0}+\sum_{k^{\prime}=0}^{n^{\prime}} \sum_{l^{\prime}=1}^{\infty} \sum_{j=0}^{m} \tau_{x z}^{j, k^{\prime}, l}\right] H\left(v_{0}-v_{\mathrm{s}}\right),
\end{aligned}
$$




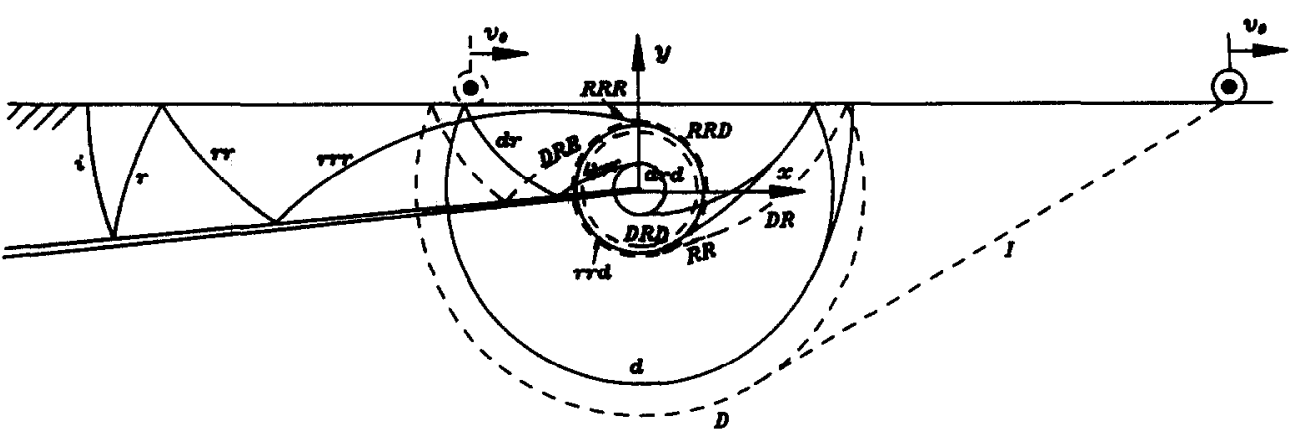

Fig. 3. Wave fronts of the incident, reflected and diffracted waves for a short time period after the dynamic loading is applied on the half-space.

$w=\sum_{i=0}^{s} w^{i}+\sum_{k=0}^{n} \sum_{l=0}^{\infty} \sum_{j=0}^{m} w^{j, k, l}+\left[\sum_{i^{\prime}=0}^{s^{\prime}} w^{i^{\prime}}+\sum_{k^{\prime}=0}^{n^{\prime}} \sum_{j=0}^{m} w^{j, k^{\prime}, 0}+\sum_{k^{\prime}=0}^{n^{\prime}} \sum_{r=1}^{\infty} \sum_{j=0}^{m} w^{j, k^{\prime}, r}\right] H\left(v_{0}-v_{\mathrm{s}}\right)$.

For the subsonic case, the transient solutions only consist of the first two terms in eqn (28). The first term with one summation is the contributions of incident cylindrical wave and reflected waves which are only generated by reflected waves, i.e. $i=0$ for incident cylindrical wave, $i=1$ for $r$ wave, $i=2$ for $r r$ wave, $i=3$ for $r r$ wave. The explicit forms are expressed as follows

$$
\begin{gathered}
\tau_{y z}^{i}=(-1)^{i} \frac{Q\left(-v_{0} t^{2} \sin \gamma \phi-v_{0} b^{2} r_{i}^{2} \sin \omega \cos \theta_{i}+r_{i} t \sin \theta_{i}\right)}{\pi\left(t^{2}-b^{2} r_{i}^{2}\right)^{1 / 2}\left(r_{i}^{2}-2 r_{i} v_{0} t \cos \omega-v_{0}^{2} b^{2} r_{i}^{2} \sin ^{2} \omega+v_{0}^{2} t^{2}\right)} H\left(t-b r_{i}\right), \\
\tau_{x z}^{i}=\frac{Q\left(v_{0} t^{2} \cos \gamma \phi-v_{0} b^{2} r_{i}^{2} \sin \omega \sin \theta_{i}-r_{i} t \cos \theta_{i}\right)}{\pi\left(t^{2}-b^{2} r_{i}^{2}\right)^{1 / 2}\left(r_{i}^{2}-2 r_{i} v_{0} t \cos \omega-v_{0}^{2} b^{2} r_{i}^{2} \sin ^{2} \omega+v_{0}^{2} t^{2}\right)} H\left(t-b r_{i}\right), \\
w^{i}=\frac{Q}{\pi \mu} \int_{0}^{t} \frac{\left(r_{i}^{2}-r_{i} v_{0} t \cos \omega\right) H\left(t-b r_{i}\right)}{\left(t^{2}-b^{2} r_{i}^{2}\right)^{1 / 2}\left(r_{i}^{2}-2 r_{i} v_{0} t \cos \omega-v_{0}^{2} b^{2} r_{i}^{2} \sin ^{2} \omega+v_{0}^{2} t^{2}\right)} \mathrm{d} t,
\end{gathered}
$$

where

$$
\begin{gathered}
\gamma=i, \quad i=0,2,4,6, \ldots \quad \gamma=i+1, \quad i=1,3,5, \ldots \\
r_{i}=\left(\left(x-l_{i}\right)^{2}+\left(y-h_{i}\right)^{2}\right)^{1 / 2}, \quad \theta_{i}=\cos ^{-1}\left(\frac{x-l_{i}}{r_{i}}\right), \quad \omega=\theta_{i}-\gamma \phi, \\
l_{0}=l, \quad h_{0}=h, \\
l_{i}=l \cos (i \phi)+h \sin (i \phi)+\sum_{m=0}^{i / 2-1} 2 h \sin (2 m \phi), \quad i=2,4,6, \ldots \\
h_{i}=-l \sin (i \phi)+h \cos (i \phi)+\sum_{m=0}^{i / 2-1} 2 h \cos (2 m \phi), \quad i=2,4,6, \ldots \\
l_{i}=l \cos ((i+1) \phi)+h \sin ((i+1) \phi)+\sum_{m=1}^{(i-1) / 2} 2 h \sin (2 m \phi), \quad i=1,3,5,7, \ldots \\
h_{i}=l \sin ((i+1) \phi)-h \cos ((i+1) \phi)-\sum_{m=1}^{(i-1) / 2} 2 h \cos (2 m \phi), \quad i=1,3,5,7, \ldots
\end{gathered}
$$

The second term in eqn (28) with three summations comes from the contributions for diffracted waves and reflected waves which are generated by diffracted waves, i.e. $k=0$, 
$l=0, j=0$ for $d$ wave, $j=1$ for $d r$ wave, $j=2$ for $d r r$ wave; $k=0, l=1, j=0$ for $d r d$ wave, $j=1$ for $d r d r$ wave, $j=2$ for $d r d r r$ wave; $k=0, l=2, j=0$ for $d r d r d$ wave, $j=1$ for $d r d r d r$ wave ; $k=1, l=0, j=0$ for $r r d$ wave, $j=1$ for $r r d r$ wave; $k=1, l=1, j=0$ for $r r d r d$ wave, $j=1$ for $r r d r d r$ wave. $k=0$ can be seen here to consist of the diffracted $d$ wave which is diffracted by the incident wave and the sequence of diffracted waves and reflected waves which are generated by the $d$ wave. $k=1$ consists of the diffracted $r r d$ wave which is diffracted from the $r r$ wave by the crack tip and the sequence of diffracted and reflected waves which are generated by the $r r d$ wave. The index notation $j=0$ will stand for the diffracted waves and $j \neq 0$ stands for the reflected waves. The detailed results are expressed in Appendix A.

However, for the supersonic case $\left(v_{0}>v_{\mathrm{s}}\right)$, the incident waves generated by the moving loading consist of not only a cylindrical wave but also a plane wave (Fig. 1). We have already expressed the complete expressions of the reflected and diffracted waves by an incident cylindrical wave in eqn (29) and Appendix A. The reflected and diffracted waves generated by the incident plane wave are expressed in eqn (28) for the last three terms. The term with one summation is the solution of incident plane wave and reflected waves which are generated by pure reflected waves, i.e. $i^{\prime}=0$ for incident plane wave, $i^{\prime}=1$ for $R$ wave, $i^{\prime}=2$ for $R R$ wave, $i^{\prime}=3$ for $R R R$ wave and all these waves are plane waves. The explicit forms can be expressed as follows

$$
\begin{gathered}
\tau_{y z}^{i}=(-1)^{i} \frac{Q}{v_{0}} \frac{\sqrt{b^{2} v_{0}^{2}-1} \cos \gamma \phi+\sin \gamma \phi}{\sqrt{b^{2} v_{0}^{2}-1}} \delta\left(t-t_{i}^{*}\right), \\
\tau_{x z}^{i^{\prime}}=\frac{Q}{v_{0}} \frac{\sqrt{b^{2} v_{0}^{2}-1} \sin \gamma \phi-\cos \gamma \phi}{\sqrt{b^{2} v_{0}^{2}-1}} \delta\left(t-t_{i}^{*}\right), \\
w^{i}=\frac{Q}{\mu \sqrt{b^{2} v_{0}^{2}-1}} H\left(t-t_{i}^{*}\right),
\end{gathered}
$$

where

$$
\begin{gathered}
\gamma=i^{\prime}+1 \quad i^{\prime}=1,3,5 \ldots \quad \gamma=i^{\prime} \quad i^{\prime}=0,2,4,6 \ldots \\
t_{0}^{*}=c(x-l)-\sqrt{b^{2}-c^{2}}(y-h), \\
t_{i}^{*}=\sqrt{b^{2}-c^{2}}\left[(-1)^{t^{\prime-1}} y_{i^{\prime}-1} \cos \phi-x_{i^{\prime}-1} \sin \phi+h\right]+c\left[x_{i^{\prime}-1} \cos \phi+(-1)^{i^{\prime}-1} y_{i^{\prime}-1} \sin \phi-l\right] .
\end{gathered}
$$

The term with two summations (i.e. $l^{\prime}=0$ ) comes from the contributions for waves which are diffracted by the crack tip only once, i.e. $k^{\prime}=0, j=0$ for $D$ wave, $j=1$ for $D R$ wave, $j=2$ for $D R R$ wave ; $k^{\prime}=1, j=0$ for $R R D$ wave, $j=1$ for $R R D R$ wave, $j=2$ for $R R D R R$ wave; $k^{\prime}=2, j=0$ for $R R R R D$ wave, $j=1$ for $R R R R D R$ wave. The correspondent results are expressed in Appendix B.

Finally, the term with three summations comes from the contributions for waves which are diffracted from the crack tip at least twice. We have $k^{\prime}=0, l^{\prime}=1, j=0$ for $D R D$ wave, $j=1$ for $D R D R$ wave, $j=2$ for $D R D R R$ wave; $k^{\prime}=0, l^{\prime}=2, j=0$ for $D R D R D$ wave, $j=1$ for $D R D R D R$ wave $k^{\prime}=1, l^{\prime}=1, j=0$ for $R R D R D$ wave, $j=1$ for $R R D R D R$ wave. The complete results are expressed in Appendix $C$.

The number of reflected waves (i.e. $n, s, m, n^{\prime}$ and $s^{\prime}$ ) is dependent on the location and the inclined angle $\phi$ of the crack, the speed of the moving load and the position where the dynamic point loading is applied. The results are expressed as follows

$$
\begin{aligned}
& \text { If } \phi_{2 j+1}<\pi / 2<\phi_{2 j-1}, \quad \text { then } n=j ; \\
& \text { If } v \phi<\phi_{1}<(v+1) \phi, \quad \text { then } \quad s=v+2
\end{aligned}
$$




$$
\text { If } v \phi<\pi / 2<(v+1) \phi \text {, then } m=v+1 \text {, }
$$

where

$$
\begin{gathered}
\phi_{2 j+1}=\cos ^{-1}\left(\frac{l_{2 j+1}}{R_{2 j+1}}\right), \\
R_{2 j+1}=\left(l_{2 j+1}^{2}+h_{2 j+1}^{2}\right)^{1 / 2}, \\
l_{2 j+1}=l \cos 2(j+1) \phi+h \sin 2(j+1) \phi+\sum_{p=1}^{n} 2 h \sin (2 p \phi), \\
\text { If } \pi-\cos ^{-1}\left(\frac{l_{2 k}}{R_{2 k}}\right)<\cos ^{-1}\left(\frac{c}{v_{0}}\right)+2 k \phi<\frac{\pi}{2} \text { and } \cos ^{-1}\left(\frac{c}{v_{0}}\right) \\
\text { If } \pi-\cos ^{-1}\left(\frac{l_{2 k}}{R_{2 k}}\right) \geqslant \cos ^{-1}\left(\frac{c}{v_{0}}\right)+2 k \phi \quad \text { then } s^{\prime}=2 k^{*}, k^{*} \text { is the maximum value of } k ; \\
\text { If } \pi-\cos ^{-1}\left(\frac{l_{2 k}}{R_{2 k}}\right)<\cos ^{-1}\left(\frac{c}{v_{0}}\right)+2 k \phi \quad \text { and } \cos ^{-1}\left(\frac{c}{v_{0}}\right)+(2 k+2) \phi \geqslant \frac{\pi}{2}, \quad \text { then } n^{\prime}=k ; \\
\text { and } \pi-(t+1) \phi<\cos ^{-1}\left(\frac{c}{v_{0}}\right)<\pi-t \phi<\pi / 2 \quad \text { then } s^{\prime}=t,
\end{gathered}
$$

where

$$
\begin{gathered}
l_{0}=l, \quad h_{0}=h, \\
l_{2 k}=l \cos (2 k \phi)+h \sin (2 k \phi)+\sum_{m=0}^{k-1} 2 h \sin 2 m \phi, \quad k=1,2,3, \ldots \\
h_{2 k}=-l \sin (2 k \phi)+h \cos (2 k \phi)+\sum_{m=0}^{k-1} 2 h \cos 2 m \phi, \quad k=1,2,3, \ldots \\
R_{2 k}=\left(l_{2 k}^{2}+h_{2 k}^{2}\right)^{1 / 2} .
\end{gathered}
$$

\section{NUMERICAL RESULTS OF TRANSIENT SOLUTIONS}

The geometric configuration considered in this study is an inclined semi-infinite crack located under the surface of a half-plane. For the subsonic case, the incident wave generated by the dynamic moving antiplane loading will be diffracted from the crack tip and reflected from the crack surface as $d$ and $r$ waves, which will be reflected from the half-plane and interact with the inclined crack again at a later time. These waves will reflect back and forth between the half-plane and the crack, which will generate an infinite number of reflected and diffracted waves. 
The complicated exact full-field solutions have been determined in the previous section in a compact form and will be used for numerical calculation. The transient response to a dynamic moving loading will be investigated numerically here with a Heaviside function $H(t)$ time dependence and with unit magnitude applied at $(-25,10)$ and moving towards the positive $x$-direction with subsonic speed. The inclined angle $\phi$ of the crack is chosen to be $30^{\circ}$. It is worthy to note that only the incident wave and the second reflected $r r$ wave will pass through the crack tip and generate a sequence of diffracted and reflected waves; the other pure reflected waves (i.e. $r r r, r r r r$, etc.) will only reflect back and forth between the crack face and the half-plane surface. Hence, in this particular case, $k$ is equal to 0 and 1 , and the associated wave fronts over a short time period are shown in Fig. 4. The field points located at $r=5, \theta=30^{\circ}, 60^{\circ}$ and $120^{\circ}$ are selected for analyzing the transient response for different moving speeds of the loading, where $r$ and $\theta$ are polar coordinates defined from the $x$-axis. The transient shear stresses of these points are plotted in Figs 5-7. The time has been normalized by dividing $b R_{0}$ where $R_{0}$ is the distance from the applied load and the crack tip. The corresponding static values for zero moving speed are also indicated in these figures. The incident and purely reflected waves are singular at their wave fronts while the diffracted waves have finite jumps at their wave fronts. For these particular field points we have investigated, the arrival times for $r r d$ and $d r d$ waves is nearly the same, which is also indicated in Fig. 4. In these figures the transient stresses for the zero moving speed of the loading tend toward the corresponding static value after the first few waves have passed the field points. The transient shear stresses $\tau_{x z}$ for these material points are plotted in Figs 8-10. The numerical results plotted in Figs 5-10 reveal that the stress shows a dynamic overshoot as compared to the corresponding quasi-static loading. It is noted that the magnitude of the dynamic overshoot increases as the moving speed increases.

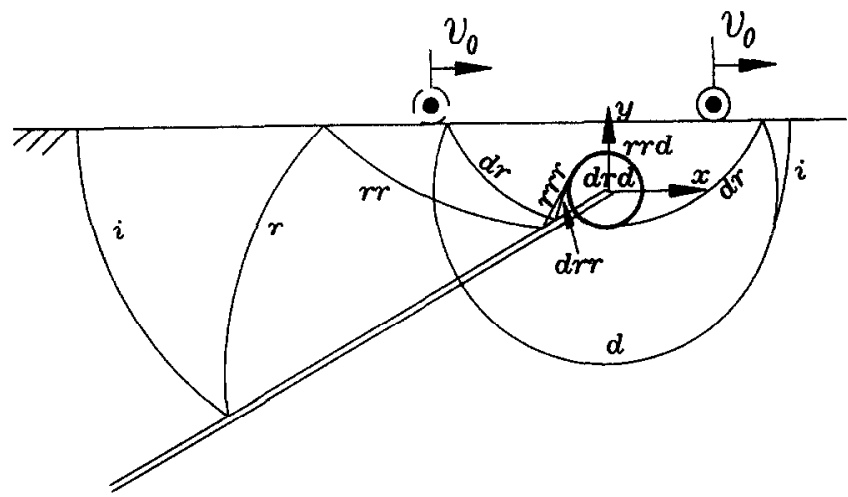

Fig. 4. The configuration of the wave fronts for a short time period after the dynamic loading is applied on the half-space and moving with subsonic speed.

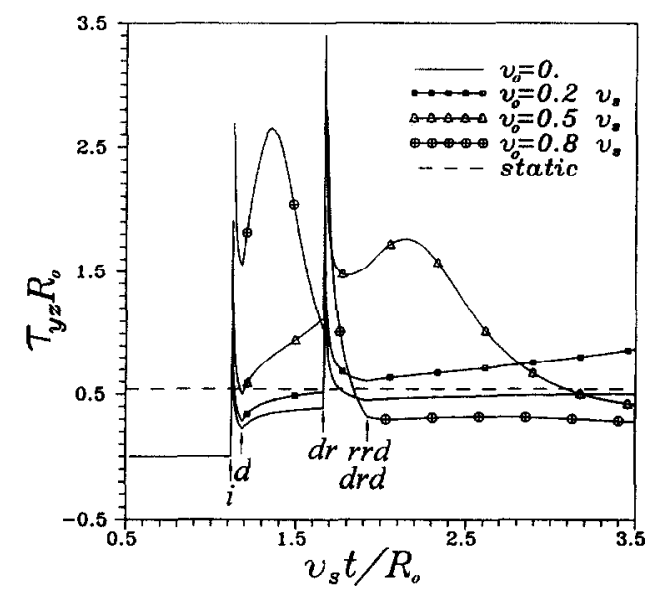

Fig. 5. Transient shear stress $\tau_{y z}$ for the field point located at $r=5, \theta=30^{\circ}$ due to impact loading applied at $(-25,10)$ and moving with subsonic speed. 


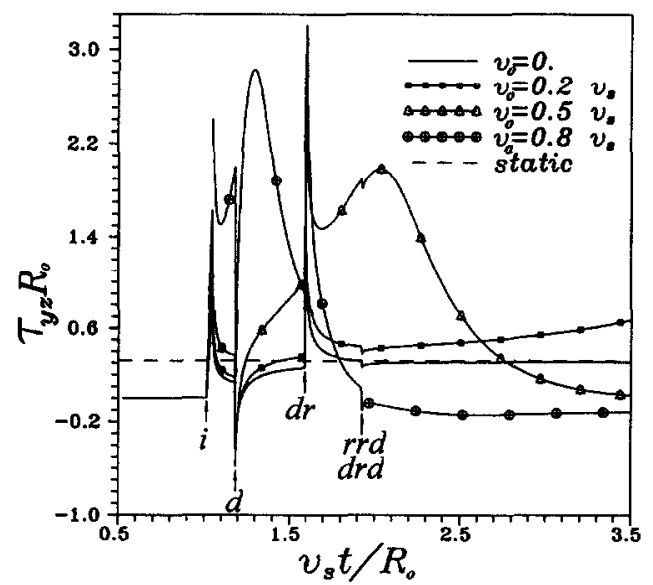

Fig. 6. Transient shear stress $\tau_{y z}$ for the field point located at $r=5, \theta=60^{\circ}$ due to impact loading applied at $(-25,10)$ and moving with subsonic speed.

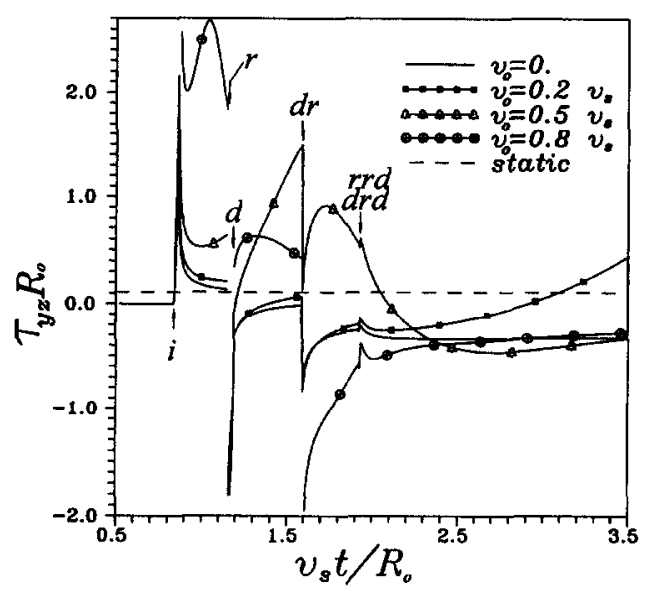

Fig. 7. Transient shear stress $\tau_{y z}$ for the field point located at $r=5, \theta=120^{\circ}$ due to impact loading applied at $(-25,10)$ and moving with subsonic speed.

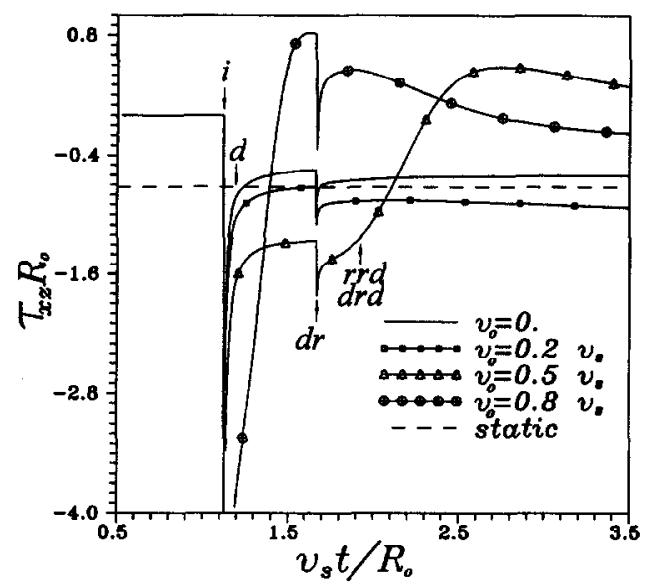

Fig. 8. Transient shear stress $\tau_{x z}$ for the field point located at $r=5, \theta=30^{\circ}$ due to impact loading applied at $(-25,10)$ and moving with subsonic speed.

\section{CONCLUSIONS}

Most of the problems which have been studied in the development of fracture mechanics are quasi-static. Because of loading conditions and material properties, numerous problems have existed for which the assumption of quasi-static deformation is invalid, and the inertia of the material must be taken into account. The propagation of stress waves 


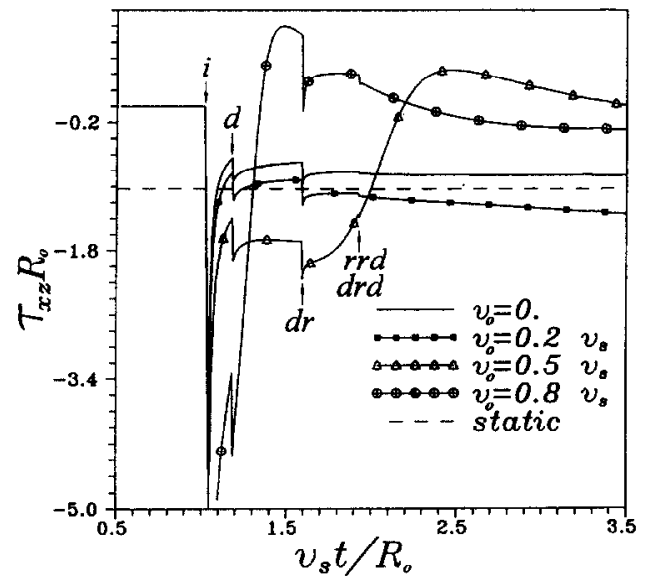

Fig. 9. Transient shear stress $\tau_{x i}$ for the field point located at $r=5, \theta=60^{\circ}$ due to impact loading applied at $(-25,10)$ and moving with subsonic speed.

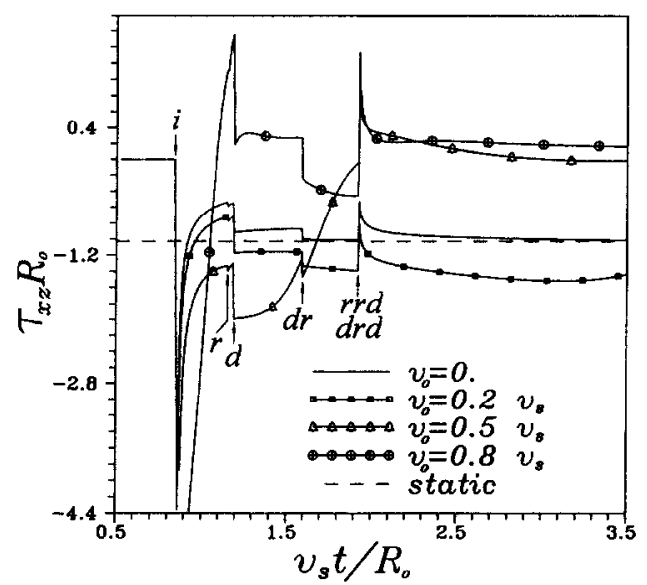

Fig. 10. Transient shear stress $\tau_{x z}$ for the field point located at $r=5, \theta=120^{\circ}$ due to impact loading applied at $(-25,10)$ and moving with subsonic speed.

through an unbounded medium is not a difficult subject. However, if boundaries are introduced, reflected and diffracted waves will be generated from boundaries, making the problem much more complicated. The problem of the diffraction of elastic waves by an internal crack is of considerable importance in a variety of engineering applications. In fracture mechanics the interest is in the determination of the stress field near the crack tip in order to study the phenomenon of crack propagation under dynamic loading.

The transient response of a half-space containing a subsurface inclined crack has been considered in this study to gain understanding of the interaction of stress waves with material defects. This problem contains a characteristic length and is solved by superposition of proposed fundamental solutions in the Laplace transform domain. The exact transient full-field solutions for stresses and displacement over a long period of time are obtained in this study. The complicated closed form for transient solutions is expressed in a compact form which accounts for all contributions coming from incident, reflected and diffracted waves generated from the crack and the half-plane boundary. The presented transient solutions are valid for both subsonic and supersonic cases, but the numerical calculations are only investigated for the subsonic case. For the special case of the moving speed of the dynamic loading equal to zero, the transient solution will approach the correspondent static value after the first few diffracted waves have passed the field points. The numerical result for the dynamic analysis shows an overshoot and the magnitude of the dynamic overshoot increases as the moving speed increases. It is, therefore, possible that crack propagation does not occur under static loading, but the material may fracture when the same system of loads is rapidly applied and generates waves. 
Acknowledgement-The research support of the National Science Council, Republic of China, through Grant NSC 83-0401-E002-115 at National Taiwan University is gratefully acknowledged.

\section{REFERENCES}

Achenbach, J. D. (1973). Wave Propagation in Elastic Solids, Elsevier, New York.

Achenbach, J. D. and Brind, R. J. (1981). Scattering of surface waves by a sub-surface crack. J. Sound Vibration 76, 43-56.

Brind, R. J. and Achenbach, J. D. (1981). Scattering of longitudinal and transverse waves by a sub-surface crack. $J$. Sound Vibration 76, 555-563.

Brock, L. M. (1982). Shear and normal impact loadings on one face of a narrow slit. Int. J. Solids Structures 18, 467-477.

Brock, L. M. (1984). Stresses in a surface obstacle undercut due to rapid indentation. J. Elasticity 14, 415-424.

Brock, L. M., Jolles, M. and Schroedl, M. (1985). Dynamic impact over a subsurface crack: applications to the dynamic tear test. ASME J. Appl. Mech. 52, 287-290.

Brock, L. M. (1986). Transient dynamic green's functions for a cracked plane. Quart. Appl. Math. XLIV, 265275.

Cagniard, L. (1958). Reflection and Refraction of Progressive Seismic Waves (Translated by E. A. Flinn and C. H. Dix), McGraw-Hill, New York.

de Hoop, A. T. (1958). Representation theorems for the displacement in an elastic solid and their application to elastodynamic diffraction theory. Doctoral dissertation, Technische Hoegschool, Delft.

Freund, L. B. (1974). The stress intensity factor due to normal impact loading of the faces of a crack. Int. $J$. Engng Sci. 12, 179-189.

Gakenheimer, D. C. and Miklowitz, J. (1969). Transient excitation of an elastic-half space by a point load traveling on the surface. ASME J. Appl. Mech. 36, 505-515.

Lee, Y. J. and Freund, L. B. (1990). Fracture initiation due to asymmetric impact loading of an edge cracked plate. ASME J. Appl. Mech. 57, 104-111.

Ma, C. C. and Chen, S. K. (1993). Exact transient analysis of an anti-plane semi-infinite crack subjected to dynamic body forces. Wave Motion 17, 161-171.

Ma, C. C. and Chen, S. K. (1994). Exact transient full field analysis of an antiplane subsurface crack subjected to dynamic impact loading. ASME J. Appl. Mech. 61, 649-655.

Ma, C. C. and Hou, Y. C. (1990). Theoretical analysis of the transient response for a stationary inplane crack subjected to dynamic impact loading. Int. J. Engng Sci. 28, 1321-1329.

Ma, C. C. and Hou, Y. C. (1991). Transient analysis for antiplane crack subjected to dynamic loadings. ASME J. Appl. Mech. 58, 703-709.

Noble, B. (1958). The Wiener-Hopf Technique, Pergamon Press, Oxford.

Payton, R. G. (1964). An application of the dynamic Betti-Rayleigh reciprocal theorem to moving point loads in elastic media. Quart. Appl. Math. 21, 299-313.

Tsai, C. H. and Ma, C. C. (1992). Transient analysis of a semi-infinite crack subjected to dynamic concentrated forces. ASME J. Appl. Mech. 59, 804-811.

\section{APPENDIX A}

The detailed results for the second term in eqn (28) are expressed as follows

$$
\begin{gathered}
\tau_{y z}^{i, k, l}=\frac{i^{a} Q}{2 \pi^{2}(2 \pi i)^{l}} \int_{b R_{0,2 k}}^{a_{1}} \int_{2 h b}^{a_{2}} \ldots \int_{2 h b}^{a_{i+1}} F U N 1_{j, k, l} \mathrm{~d} t_{k+l+1} \mathrm{~d} t_{k+1} \ldots \mathrm{d} t_{k+1}, \\
\tau_{x z}^{j, k, l}=\frac{-Q}{2 \pi^{2}(2 \pi)^{\prime}} \int_{b R_{0,2 k}}^{a_{1}} \int_{2 h b}^{a_{2}} \ldots \int_{2 h b}^{a_{l+1}} F U N 2_{j, k, l} \mathrm{~d} t_{k+l+1} \mathrm{~d} t_{k+1} \ldots \mathrm{d} t_{k+1}, \\
w^{j, k, l}=\frac{-Q}{2 \pi^{2}(2 \pi)^{l} \mu} \int_{b k}^{t} \int_{b R_{0,2 k}}^{a_{1}^{\prime}} \int_{2 h b}^{a_{2}^{\prime}} \cdots \int_{2 h b}^{a_{i+1}^{\prime}} F U N 3_{j, k, l} \mathrm{~d} t_{k+l+1} \mathrm{~d} t_{k+l} \ldots \mathrm{d} t_{k+1} \mathrm{~d} \tau,
\end{gathered}
$$

where

$$
\begin{gathered}
b k=b\left(R_{0,2 k}+d_{j}+2 l h\right), \\
a_{1}=t-b d_{j}-2 l h b, \quad a_{1}^{\prime}=\tau-b d_{j}-2 l h b, \\
a_{v+1}=t-b d_{j}-t_{1}-t_{2}-\cdots-t_{v}-2(l-v) h b, \quad v=1,2,3 \ldots, l \\
a_{v+1}^{\prime}=\tau-b d_{j}-t_{1}-t_{2}-\cdots-t_{v}-2(l-v) h b, \quad v=1,2,3 \ldots, l
\end{gathered}
$$




$$
\begin{gathered}
F U N 2_{j, k, l}=O p\left[\frac{S_{1, l+k+p+2}^{+}}{\left(b^{2}-S_{1, l+k+p+2}^{1}\right)^{1 / 2}} \frac{G\left(S_{2, k+1}^{ \pm}, \eta_{k+2}^{ \pm}\right)}{1+v_{0}\left(\eta_{k+1}^{\perp} \cos \phi-\alpha_{k+1}^{ \pm} \sin \phi\right)} G\left(S_{2, k+2}^{ \pm}, \eta_{k+3}^{ \pm}\right) \ldots\right. \\
\left.G\left(S_{2, k, l 11}^{ \pm}, \eta_{k+1,2}^{+}\right)\left( \pm \frac{\partial S_{2, k+1}^{ \pm}}{\partial t_{k+1}}\right)\left( \pm \frac{\partial S_{2, k+2}^{ \pm}}{\partial t_{k+2}}\right) \ldots\left( \pm \frac{\partial S_{2, k+1+1}^{ \pm}}{\partial t_{k+1+1}}\right)\left(\frac{\partial S_{1, k+1+p+2}^{+}}{\partial t_{k+l+p+2}}\right)\right], \\
F U N 3_{j, k, l}=O p\left[\frac{1}{\left(b^{2}-S_{1, l+k+p+2}^{+2}\right)^{1 / 2}} \frac{G\left(S_{2, k+1}^{ \pm}, \eta_{k+2}^{ \pm}\right)}{1+v_{0}\left(\eta_{k+1}^{ \pm} \cos \phi-\alpha_{k+1}^{ \pm} \sin \phi\right)} G\left(S_{2, k+2}^{ \pm}, \eta_{k+3}^{ \pm}\right) \ldots\right. \\
\left.G\left(S_{2, k+1+1}^{ \pm}, \eta_{k+1+2}^{+}\right)\left( \pm \frac{\partial S_{2, k+1}^{ \pm}}{\partial t_{k+1}}\right)\left( \pm \frac{\partial S_{2, k+2}^{ \pm}}{\partial t_{k+2}}\right) \ldots\left( \pm \frac{\partial S_{2, k+l+1}^{ \pm}}{\partial t_{k+1+1}}\right)\left(\frac{\partial S_{1, k+1+p+2}^{+}}{\partial t_{k+1+p+2}}\right)\right],
\end{gathered}
$$

in which the operator "Op" is "Re" or "Im" depending on whether $l$ is even or odd and

$$
\begin{aligned}
& S_{1, k+1+p+2}^{+}=-\frac{t_{k+1+p+2} \cos \psi_{j}^{\prime}}{s_{f}}+i \frac{\sin \psi_{j}^{\prime}}{s_{j}}\left(t_{k+1+p+2}^{2}-b^{2} d_{j}^{2}\right)^{1 / 2}, \\
& S_{2, k+1}^{+}=\frac{t_{k+1} \cos \Theta_{0,2 k}}{R_{0,2 k}} \pm i \frac{\sin \Theta_{0,2 k}}{R_{0,2 k}}\left(t_{k+1}^{2}-b^{2} R_{0,2 k}^{2}\right)^{1 / 2} \text {, } \\
& S_{2, k+v+1}^{+}=\frac{t_{k+v+1} \sin \phi}{2 h} \pm i \frac{\cos \phi}{2 h}\left(t_{k+v+1}^{2}-4 b^{2} h^{2}\right)^{1 / 2}, \quad v=1,2,3, \ldots, l \\
& \eta_{k+l+2}^{+}=-\frac{t_{k+l+p+2} \cos \psi_{j}}{d_{j}}+i \frac{\sin \psi_{j}}{d_{j}}\left(t_{k+1+p+2}^{2}-b^{2} d_{j}^{2}\right)^{1 / 2} \\
& \eta_{k+v+1}^{ \pm}=-\frac{t_{k+v+1} \sin \phi}{2 h} \pm i \frac{\cos \phi}{2 h}\left(t_{k+v+1}^{2}-4 b^{2} h^{2}\right)^{1 / 2}, \quad v=1,2,3, \ldots, l \\
& S_{2,1}=\eta_{1}, \quad t_{k+1+p+2}=t-t_{k+1}-t_{k+2}-\cdots-t_{k+1+1}, \\
& q=0, \quad \text { when } l=0,2,4,6, \ldots ; \quad q=1, \quad \text { when } l=1,3,5,7, \ldots \\
& p=j / 2, \quad \text { when } j=0,2,4,6, \ldots ; \quad p=(j-1) / 2, \quad \text { when } j=1,3,5,7, \ldots \\
& R_{\mathbf{0}, 2 k}=\left(l_{0,2 k}^{2}+h_{0,2 k}^{2}\right)^{1 / 2} ; \quad d_{j}=\left(x_{j}^{2}+y_{j}^{2}\right)^{1 / 2}, \\
& s_{j}=\left(\left(x-l_{j}\right)^{2}+\left(y-h_{j}\right)^{2}\right)^{1 / 2} ; \quad \cos \psi_{j}^{\prime}=\frac{x-l_{j}}{s_{j}}, \\
& \cos \psi_{i}=\frac{x_{j}}{d_{j}} ; \quad \cos \Theta_{0,2 k}=\frac{l_{0,2 k}}{R_{0,2 k}}, \\
& l_{0.2 k}=l \cos (2 k+1) \phi+h \sin (2 k+1) \phi+\sum_{m=0}^{k-1} 2 h \sin (2 m+1) \phi \\
& h_{0,2 k}=-l \sin (2 k+1) \phi+h \cos (2 k+1) \phi+\sum_{m \rightarrow 0}^{k-1} 2 h \cos (2 m+1) \phi, \\
& x_{j}=\left(x-l_{j}\right) \cos (j+1) \phi+\left(y-h_{j}\right) \sin (j+1) \phi, j=0,2,4,6, \ldots \\
& y_{j}=-\left(x-l_{j}\right) \sin (j+1) \phi+\left(y-h_{j}\right) \cos (j+1) \phi, \quad j=0,2,4,6, \ldots \\
& x_{j}=\left(x-l_{j}\right) \cos (j \phi)-\left(y-h_{j}\right) \sin (j \phi), \quad j=1,3,5,7, \ldots \\
& y_{j}=\left(x-l_{j}\right) \sin (j \phi)+\left(y-h_{j}\right) \cos (j \phi), \quad j=1,3,5,7, \ldots \\
& l_{0}=0 ; \quad h_{0}=0 \text {, } \\
& l_{j}=\sum_{m=1}^{j / 2} 2 h \sin (2 m \phi), \quad j=2,4,6, \ldots \\
& h_{j}=-\sum_{m=1}^{j / 2} 2 h \cos (2 m \phi), \quad j=2,4,6, \ldots \\
& l_{j}=\sum_{m=0}^{(j-1) / 2} 2 h \sin (2 m \phi), \quad j=1,3,5,7, \ldots \\
& h_{j}=\sum_{m=0}^{(j-1) / 2} 2 h \cos (2 m \phi), \quad j=1,3,5,7, \ldots
\end{aligned}
$$




\section{APPENDIX B}

The correspondent results for the term with two summations in eqn (28) are (i.e. the supersonic case $v_{0}>v_{s}$ and $\left.l^{\prime}=0\right)$

$$
\begin{aligned}
& \tau_{y z}^{j, k^{\prime}, 0}=(-1)^{j+1} \frac{Q}{\pi} M\left(k^{\prime}\right) \operatorname{Im}\left[\frac{\left(b+\eta_{k^{+}+2}^{+}\right)}{-\cos \left(2 k^{\prime}+1\right) \phi+\sqrt{b^{2} v_{0}^{2}-1} \sin \left(2 k^{\prime}+1\right) \phi-v_{0} \eta_{k^{\prime}+2}^{+}} \frac{\partial S_{2 p+1, k^{\prime}+2}^{+}}{\partial t}\right] \\
& H\left[t-b d_{j}+c\left(l-l_{k}\right)-\sqrt{b^{2}-c^{2}}\left(h+h_{k^{\prime}}\right)\right], \\
& H\left[t-b d_{j}+c\left(l-l_{k}\right)-\sqrt{b^{2}-c^{2}}\left(h+h_{k^{\prime}}\right)\right], \\
& w^{j \cdot k^{\prime} 0}=\frac{Q}{\pi \mu} M\left(k^{\prime}\right) \int_{0}^{t} \operatorname{Im}\left[\frac{\left(b+\eta_{k^{\prime}+2}^{+}\right)}{\beta_{2 p+1, k^{\prime}+2}^{+}\left[-\cos \left(2 k^{\prime}+1\right) \phi+\sqrt{b^{2} v_{0}^{2}-1} \sin \left(2 k^{\prime}+1\right) \phi-v_{0} \eta_{k^{\prime}+2}^{+}\right]} \frac{\partial S_{2 p+1, k^{\prime}+2}^{+}}{\partial t}\right] \\
& H\left[t-b d_{j}+c\left(l-l_{k^{\prime}}\right)-\sqrt{b^{2}-c^{2}}\left(h+h_{k^{\prime}}\right)\right] \mathrm{d} t,
\end{aligned}
$$

where

$$
\begin{gathered}
l_{0}=0, \quad h_{0}=0, \\
l_{k^{\prime}}=\sum_{m=1}^{k^{\prime}} 2 h \sin (2 m \phi), \quad h_{k^{\prime}}=\sum_{m=1}^{k^{\prime}} 2 h \cos (2 m \phi), \\
M\left(k^{\prime}\right)=\frac{\sqrt{b^{2} v_{0}^{2}-1} \cos \left(2 k^{\prime}+1\right) \phi+\sin \left(2 k^{\prime}+1\right) \phi}{\sqrt{b^{2} v_{0}^{2}-1}\left[b-c \cos \left(2 k^{\prime}+1\right) \phi+\sqrt{b^{2}-c^{2}} \sin \left(2 k^{\prime}+1\right) \phi\right]^{1 / 2}}, \\
\eta_{k^{\prime}+2}^{+}=\frac{1}{d_{j}}\left\{-\left[t+c\left(l-l_{k^{\prime}}\right)-\sqrt{b^{2}-c^{2}}\left(h+h_{k^{\prime}}\right)\right] \cos \psi_{j}+i \sin \psi_{j}\left[\left(t+c\left(l-l_{k^{\prime}}\right)-\sqrt{b^{2}-c^{2}}\left(h+h_{k^{\prime}}\right)\right)^{2}-b^{2} d_{j}^{2}\right]^{1 / 2}\right\}, \\
\quad \frac{1}{d_{j}}\left\{-\left[t+c\left(l-l_{k^{\prime}}\right)-\sqrt{b^{2}-c^{2}}\left(h+h_{k^{\prime}}\right)\right] \cos \left(\psi_{j}+(2 p+1) \phi\right)\right. \\
\left.+i \sin \left(\psi_{j}+(2 p+1) \phi\right)\left[\left(t+c\left(l-l_{k^{\prime}}\right)-\sqrt{b^{2}-c^{2}}\left(h+h_{k^{\prime}}\right)\right)^{2}-b^{2} d_{j}^{2}\right]^{1 / 2}\right\}, \\
\beta_{2 p+1, k^{\prime}+2}=\left(b^{2}-S_{2 p+1, k^{\prime}+2}^{2}\right)^{1 / 2}, \\
p=j / 2, \quad \text { when } \quad j=0,2,4,6 \ldots ; \\
p=(j-1) / 2, \quad \text { when } \quad j=1,3,5,7, \ldots
\end{gathered}
$$

\section{APPENDIX C}

The detailed results for the last term with three summations in eqn (28) for the supersonic case are expressed as follows

$$
\begin{aligned}
& \tau_{y z}^{j, k^{\prime} \cdot l}=\frac{i^{q} Q M\left(k^{\prime}\right)}{2 \pi^{2}(2 \pi i)^{r-1}} \int_{2 h h}^{a_{2}} \int_{2 h b}^{a_{3}} \ldots \int_{2 h b}^{a_{i+1}} F U N 1_{j, k, l} \mathrm{~d} t_{k^{\prime}+r+1} \ldots \mathrm{d} t_{k^{\prime}+2}, \\
& \tau_{x z}^{j . k \cdot r}=\frac{-Q M\left(k^{\prime}\right)}{2 \pi^{2}(2 \pi)^{r-1}} \int_{2 h b}^{a_{2}} \int_{2 h b}^{a_{3}} \cdots \int_{2 h b}^{a_{r+1}} F U N 2_{j, k \cdot l^{\prime}} \mathrm{d} t_{k^{\prime}+\gamma^{\prime}+1} \ldots \mathrm{d} t_{k^{\prime}+2}, \\
& w^{j, k^{\prime}, l^{\prime}}=\frac{-Q M\left(k^{\prime}\right)}{2 \pi^{2}(2 \pi)^{r-1} \mu} \int_{b k^{\prime}}^{t} \int_{2 h b}^{a_{2}^{\prime}} \int_{2 h b}^{a_{3}^{\prime}} \ldots \int_{2 h b}^{a_{r+1}} F U N 3_{j, k^{\prime}, l^{\prime}} \mathrm{d} t_{k^{\prime}+t^{\prime}+1} \ldots \mathrm{d} t_{k^{\prime}+2} \mathrm{~d} \tau \text {, }
\end{aligned}
$$

where

$$
\begin{aligned}
b k^{\prime} & =\sqrt{b^{2}-c^{2}}\left(h+h_{k^{\prime}}\right)-c\left(l-l_{k^{\prime}}\right)+b\left(d_{j}+2 l^{\prime} h\right), \\
a_{2} & =t-b d_{j}-2\left(l^{\prime}-1\right) h b-\sqrt{b^{2}-c^{2}}\left(h+h_{k^{\prime}}\right)+c\left(l-l_{k^{\prime}}\right), \\
a_{2}^{\prime} & =\tau-b d_{j}-2\left(l^{\prime}-1\right) h b-\sqrt{b^{2}-c^{2}}\left(h+h_{k^{\prime}}\right)+c\left(l-l_{k^{\prime}}\right), \\
a_{t^{\prime}+1} & =t-b d_{j}-t_{2}-t_{3}-\cdots-t_{t^{\prime}}-2\left(l^{\prime}-v^{\prime}\right) h b-\sqrt{b^{2}-c^{2}}\left(h+h_{k^{\prime}}\right)+c\left(l-l_{k^{\prime}}\right), \\
v^{\prime} & =2,3,4 \ldots, l^{\prime} \\
a_{v^{\prime}+1}^{\prime} & =\tau-b d_{j}-t_{2}-t_{3}-\cdots-t_{k^{\prime}}-2\left(l^{\prime}-v^{\prime}\right) h b-\sqrt{b^{2}-c^{2}}\left(h+h_{k^{\prime}}\right)+c\left(l-l_{k^{\prime}}\right),
\end{aligned}
$$




$$
\begin{aligned}
& q=0, \text { when } \quad l^{\prime}=1,3,5, \ldots ; \\
& q=1, \text { when } \quad t^{\prime}=2,4,6, \ldots
\end{aligned}
$$

$F U N 1_{, k, t}-\mathrm{Op}\left[\frac{G\left(S_{2, k^{\prime}+2,}^{ \pm}, \eta_{k^{+}+3}^{ \pm}\right) G\left(S_{2, k^{\prime}+3}^{ \pm}, \eta_{k^{\prime}+4}^{ \pm}\right)}{-\cos \left(2 k^{\prime}+1\right) \phi+\sqrt{b^{2} v_{0}^{2}-1} \sin \left(2 k^{\prime}+1\right) \phi-v_{0} \eta_{k^{\prime}+2}^{ \pm}} \ldots\right.$

$$
\left.G\left(S_{2, k^{\prime}+r^{\prime}+1}^{ \pm}, \eta_{k^{\prime}+r^{\prime}+2}^{+}\right)\left(b+\eta_{k^{\prime}+2}^{ \pm}\right)^{1 / 2}\left( \pm \frac{\partial S_{2, k^{\prime}+2}^{ \pm}}{\partial t_{k^{\prime}+2}}\right) \ldots\left( \pm \frac{\partial S_{2, k^{\prime}+r^{\prime}+1}^{ \pm}}{\partial t_{k^{\prime}+t+1}}\right)\left(\frac{\partial S_{1, k^{\prime}+r^{\prime}+p+2}^{+}}{\partial t_{k^{\prime}+r+p+2}}\right)\right]
$$

$F U N 2_{j, k^{\prime},}=O p\left[\frac{S_{i, f+k^{\prime}+p+2}^{+} G\left(S_{2, k^{\prime}+2}^{ \pm}, \eta_{k^{\prime}+3}^{ \pm}\right)}{\beta_{1, I+k^{\prime}+p+2}^{+}\left[-\cos \left(2 k^{\prime}+1\right) \phi+\sqrt{b^{2} v_{0}^{2}-1} \sin \left(2 k^{\prime}+1\right) \phi-v_{0} \eta_{k^{\prime}+2}^{ \pm}\right]}\right.$

$$
\left.G\left(S_{2, k^{\prime}+3}^{ \pm}, \eta_{k^{+}+4}^{ \pm}\right) \ldots G\left(S_{2, k^{\prime}+l^{\prime}+1}^{ \pm}, \eta_{k^{\prime}+l^{+}+2}^{+}\right)\left(b+\eta_{k^{\prime}+2}^{ \pm}\right)^{1,2}\left( \pm \frac{\partial S_{2, k^{+}+2}^{ \pm}}{\partial t_{k^{\prime}+2}}\right) \ldots\left(\frac{\partial S_{1, k^{\prime}+l^{+}+p+2}^{+}}{\partial t_{k^{\prime}+l^{\prime}+p+2}}\right)\right],
$$

$F U N 3_{i, k^{\prime}, l^{\prime}}=\mathrm{Op}\left[\frac{G\left(S_{2, k^{\prime}+2}^{+}, \eta_{k^{\prime}+3}^{+}\right) G\left(S_{2, k^{\prime}+3}^{+}, \eta_{k^{\prime}+4}^{+}\right)}{\beta_{1, \ell^{\prime}+k^{\prime}+p^{\prime}+2}^{+}\left[-\cos \left(2 k^{\prime}+1\right) \phi+\sqrt{b^{2} v_{0}^{2}-1} \sin \left(2 k^{\prime}+1\right) \phi-v_{0} \eta_{k^{\prime}+2}^{+}\right]} \ldots\right.$

$$
\left.G\left(S_{2, k+r_{1}+1, \eta_{k^{\prime}+l+2}^{+}}^{ \pm}\right)\left(h+\eta_{k^{\prime}+2}^{ \pm}\right)^{1 / 2}\left( \pm \frac{\partial S_{2, k^{\prime}+2}^{ \pm}}{\partial t_{k^{\prime}+2}}\right) \ldots\left( \pm \frac{\partial S_{2, k^{\prime}+t^{\prime}+1}^{ \pm}}{\partial t_{k^{\prime}+t+1}}\right)\left(\frac{\partial S_{1, k^{\prime}+r^{+}+p+2}^{+}}{\partial t_{k^{\prime}+l^{\prime}+p+2}}\right)\right]
$$

in which the operator "Op" is "Re" (or "Im") if $l$ is odd (or even) and

$$
\begin{aligned}
& S_{1, k-t+p+2}^{+}=-\frac{t_{k^{\prime}+p^{\prime}+p+2} \cos \psi_{j}^{\prime}}{s_{j}}+i \frac{\sin \psi_{j}^{\prime}}{s_{j}}\left(t_{k^{\prime}+r^{\prime}+p+2}^{2}-b^{2} d_{j}^{2}\right)^{1 / 2} . \\
& \eta_{k^{+}+2}^{+}=-\frac{t_{k^{+}+2} \sin \phi}{2 h} \pm i \frac{\cos \phi}{2 h}\left(t_{k^{\prime}+2}^{2}-4 b^{2} h^{2}\right)^{1 / 2} \\
& S_{2 ., h^{\prime}+r^{\prime}+1}^{+}=\frac{t_{k^{+}+r^{\prime}+1} \sin \phi}{2 h} \pm i \frac{\cos \phi}{2 h}\left(t_{k^{\prime}+i^{\prime}+1}^{2}-4 h^{2} h^{2}\right)^{1 ; 2}, \quad t^{\prime}=1,2,3, \ldots l^{\prime} \\
& \eta_{k^{+}+r^{\prime}+2}=\frac{t_{k^{\prime}+l^{\prime}+2} \sin \phi}{2 h} \pm i \frac{\cos \phi}{2 h}\left(t_{k^{\prime}+t^{\prime}+2}^{2} \quad 4 b^{2} h^{2}\right)^{1 / 2}, \quad v^{\prime}=1,2,3, \ldots l^{\prime} \\
& \eta_{k^{+}+t+2}^{+}=-\frac{t_{k+r+p+2} \cos \psi_{j}}{d_{j}}+i \frac{\sin \psi_{j}}{d_{j}}\left(t_{k^{\prime}+r^{2}+p+2}-b^{2} d_{j}^{2}\right)^{1 / 2}, \\
& \beta_{1, k+l+p+2}^{+}=\left[b^{2}-\left(S_{1, k+l+p+2}^{+}\right)^{2}\right]^{1 / 2} .
\end{aligned}
$$

